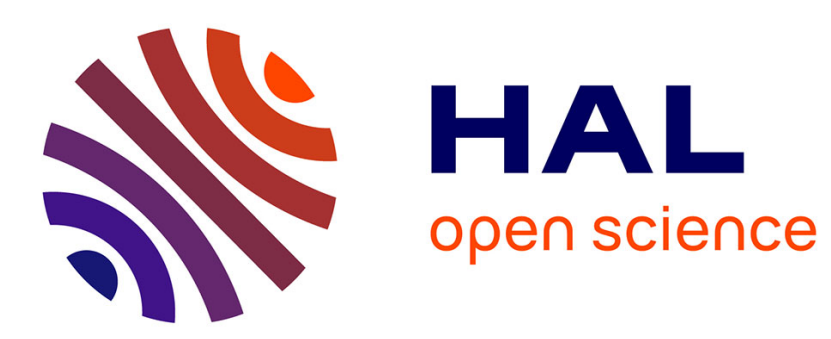

\title{
DYNAMIC CROSS-LAYER SPECTRUM ALLOCATION FOR MULTI-BAND HIGH-RATE UWB SYSTEMS
}

Ayman Khalil, Matthieu Crussière, Jean-François Hélard

\section{- To cite this version:}

Ayman Khalil, Matthieu Crussière, Jean-François Hélard. DYNAMIC CROSS-LAYER SPECTRUM ALLOCATION FOR MULTI-BAND HIGH-RATE UWB SYSTEMS. Multi-Carrier Systems and Solutions, 2009, Germany. pp.355-364, 10.1007/978-90-481-2530-2-34 . hal-00406385

\section{HAL Id: hal-00406385 \\ https://hal.science/hal-00406385}

Submitted on 22 Jul 2009

HAL is a multi-disciplinary open access archive for the deposit and dissemination of scientific research documents, whether they are published or not. The documents may come from teaching and research institutions in France or abroad, or from public or private research centers.
L'archive ouverte pluridisciplinaire HAL, est destinée au dépôt et à la diffusion de documents scientifiques de niveau recherche, publiés ou non, émanant des établissements d'enseignement et de recherche français ou étrangers, des laboratoires publics ou privés. 


\title{
DYNAMIC CROSS-LAYER SPECTRUM ALLOCATION FOR MULTI-BAND HIGH-RATE UWB SYSTEMS
}

\author{
Ayman Khalil, Matthieu Crussière and Jean-François Hélard \\ Institute of Electronics and Telecommunications of Rennes (IETR) \\ INSA, 20 avenue des Buttes de Coëmes, 35043 Rennes, France \\ ayman.khalil@insa-rennes.fr
}

\begin{abstract}
In this paper, we investigate a new approach for the spectrum allocation in UWB systems. This approach consists in a cross-layer scheme that takes into consideration the different users channel quality and quality of service (QoS) requirements. The new scheme is based on the WiMedia solution proposed for multiband OFDM UWB systems. The main objective is to propose a lowcomplexity solution for the spectrum allocation that can manage all the users constraints. Thus, we study the optimal solution of the spectrum allocation and formulate it as a convex optimization problem. Then, we show that our proposed scheme reduces significantly the complexity of the optimal solution. Moreover, we show through simulations, that the new approach and the optimal solution have close performance in term of error rate and they outperform WiMedia solution proposed for UWB systems.
\end{abstract}

Keywords - OFDM, spectrum allocation, QoS, UWB.

\section{INTRODUCTION}

Ultra-wideband transmission is an emerging technology for future highrate, short-range wireless communications. Its wide bandwidth and low transmission power density make it attractive to researchers since 2002 when the Federal Communications Commission (FCC) regulated UWB systems by allocating the 3.1 to $10.6 \mathrm{GHz}$ spectrum for unlicensed use of UWB [1]. In order to reduce interference with other existing systems, the FCC imposed a power spectral density (PSD) limit of $-41.3 \mathrm{dBm} / \mathrm{MHz}$.

The IEEE 802.15a wireless personal area networks (WPAN) standardization group defined a very high data rate physical layer based on UWB signalling. One of the multiple-access techniques considered by the group is a multiband orthogonal frequency division multiplexing (MBOFDM) supported by the MultiBand OFDM Alliance (MBOA) and the WiMedia forum [2], [3] which merged in March 2005 and are today known as the WiMedia Alliance.

On December 2005, ECMA International approved two standards for UWB technology based on the WiMedia solution: ECMA-368 for high rate 
UWB PHY and MAC standard and ECMA-369 for MAC-PHY Interface for ECMA-368 [4].

There have been a lot of studies on the resource allocation in UWB system based on the WiMedia solution. However, to this date, most research studies on multiband UWB systems have been devoted to the physical layer issues. In [5], [6], the authors propose resource allocation solutions for OFDMUWB systems in a single-user scheme. In [7], the author considers the multiuser context but without taking into consideration the users QoS requirements.

The aim of this paper is to propose a spectrum management scheme based on UWB signalling in a multiple medium access demand to ensure dynamic spectrum utilization that differentiates between existing users. Therefore, we propose to classify the users into two classes, the first called QoS class for real-time applications that have strict QoS requirements (video recording, $\mathrm{A} / \mathrm{V}$ conferencing, interactive gaming, etc). The second class is called best effort (BE) class for non real-time applications that have tolerance to some QoS requirements (file transfer, Internet, etc). On the other hand, we propose to represent each user channel quality by exploiting the exponential effective SINR mapping (EESM) method used in OFDM systems [8]. Consequently, we formulate the resource allocation problem as a convex optimization problem taking into account the users classification and the EESM method issues. Then, we propose our cross-layer approach that reduces the complexity of the optimal solution given by the convex optimization problem.

This paper is organized as follows. Section 2 introduces the WiMedia model by presenting the PHY and MAC layers characteristics. Section 3 derives the problem formulation as a convex optimization problem and presents the optimal solution. In section 4, we give the proposed lowcomplexity cross-layer solution. Section 5 presents simulation results showing the comparison between the proposed scheme and the optimal solution, and the performance of the multiuser solution compared to the single-user WiMedia solution. Finally, section 6 concludes this paper.

\section{SYSTEM MODEL}

\subsection{PHY Layer}

The WiMedia solution consists in combining OFDM with a multi-banding technique that divides the available band into 14 sub-bands of $528 \mathrm{MHz}$, as illustrated in Fig. 1. An OFDM signal can be transmitted on each sub-band using a 128-point inverse fast Fourrier transform (IFFT). Out of the 128 subcarriers used, only 100 are assigned to transmit data. Different data rates 


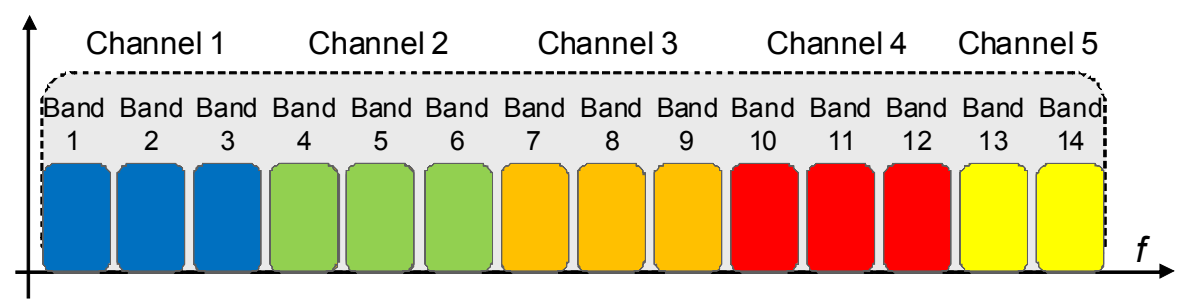

Figure 1. Channel distribution for WiMedia solution.

from 53.3 to $480 \mathrm{Mbit} / \mathrm{s}$ are obtained through the use of forward error correction (FEC), frequency-domain spreading (FDS) and time-domain spreading (TDS), as presented in Table 1. The constellation applied to the different subcarriers is either a quadrature phase-shift keying (QPSK) for the low data rates or a dual carrier modulation (DCM) for the high data rates. Time-frequency codes (TFC) are used to provide frequency hopping from a sub-band to another at the end of each OFDM symbol. TFC allows every user to benefit from frequency diversity over a bandwidth equal to the three sub-bands of one channel. In addition, to prevent from interference between consecutive symbols, a zero padding (ZP) guard interval is inserted instead of the traditional cyclic prefix (CP) used in the classical OFDM systems [10].

The WiMedia solution offers potential advantages for high-rate UWB applications, such as the signal robustness against channel selectivity and the efficient exploitation of the energy of every signal received within the prefix margin. However, we will see in the next section that the exploitation of the PHY layer at the MAC level is suboptimal in a multi-user context since the medium access mechanisms do not take advantage of the sub-band structure.

Table 1

WiMedia system data rates

\begin{tabular}{ccccc}
\hline $\begin{array}{c}\text { Data } \\
\text { Rate } \\
\text { (Mbit/s })\end{array}$ & Modulation & $\begin{array}{c}\text { Coding } \\
\text { Rate }\end{array}$ & FDS & TDS \\
\hline 53.3 & QPSK & $1 / 3$ & Yes & Yes \\
80 & QPSK & $1 / 2$ & Yes & Yes \\
110 & QPSK & $11 / 32$ & No & Yes \\
160 & QPSK & $1 / 2$ & No & Yes \\
200 & QPSK & $5 / 8$ & No & No \\
320 & DCM & $1 / 2$ & No & No \\
400 & DCM & $5 / 8$ & No & No \\
480 & DCM & $3 / 4$ & No & No \\
\hline
\end{tabular}




\subsection{MAC Layer}

The WiMedia MAC protocol is a distributed TDMA-based MAC protocol as defined in ECMA standard. Time is divided into superframes where each frame is composed of 256 medium access slots (MAS).

Each MAS has a length of $256 \mu \mathrm{s}$. Each superframe starts with a beacon period (BP) that is responsible for the exchange of reservation information, the establishment of neighbourhood information and many other functions.

WiMedia defines two access mechanisms: the prioritized contention access (PCA) and the distributed reservation protocol (DRP). PCA provides differentiated access to the medium for four access categories (ACs); it is similar to the enhanced distributed channel access (EDCA) mechanism of IEEE 802.11e standard. On the other hand, DRP is a TDMA-based mechanism which enables a device to reserve one or more MASs for the communication with neighbours.

\subsection{Channel Model}

The channel model used in this study is the one adopted by IEEE 802.15.3a committee for the evaluation of UWB proposals [11]. It is a modified version of the Saleh-Valenzuela model for indoor channels, fitting the properties of measured UWB channels. A lognormal distribution is used for the multipath gain magnitude. In addition, independent fading is assumed for each cluster and each ray within the cluster.

Four different channel models (CM1 to CM4) are defined for the UWB system modelling, each with arrival rates and decay factors chosen to match different usage scenarios and to fit line-of-sight (LOS) and non-line-of-sight (NLOS) cases.

\section{OPTIMAL SPECTRUM ALLOCATION}

Our main goal is to find the best sub-band assignment for the heterogeneous users. Therefore, we propose first to represent their channel quality in each sub-band by using the EESM method. The basic idea of this method is to find a compression function that maps a sequence of varying SINRs to a single value that is strongly correlated with the actual BER [9]. In WiMedia case, one channel is divided into three sub-bands and the allocation is made by sub-band; that means that each user is dynamically allocated one sub-band for the duration of one superframe. Therefore, the effective SINR calculated for each sub-band is given by 


$$
\operatorname{SINR}_{\text {eff }}=-\lambda \ln \left(\frac{1}{N} \sum_{i=1}^{N} e^{-\frac{S I N R_{i}}{\lambda}}\right)
$$

where $\lambda$ is a scaling factor that depends on the selected modulation and coding scheme (MCS), $N$ is the number of subcarriers in a sub-band, and $\operatorname{SINR}_{i}$ is the ratio of signal to interference and noise on the $i^{\text {th }}$ sub-carrier.

In our system model, we compute the effective SINR value for each user in each sub-band by using (1). For instance, in the case of one channel divided into $N_{b}=3$ sub-bands, and with $N_{u}=3$ users, the computation result is a matrix containing $N_{b} \times N_{u}=9$ effective SINR values.

After representing the channel power by the effective SINR value, we formulate our allocation problem which goal is to maximize the BE users data rate while maintaining a certain data rate threshold for QoS users under a total power $P_{T}$ constraint.

Let $U$ is the total number of users, $U_{Q o S}$ is the QoS users number and $U_{B E}\left(U-U_{Q O S}\right)$ is the BE users number. The rate of a user $u$ in a sub-band $b$ is defined as

$$
r_{u, b}=\log _{2}\left(1+P_{u, b} \mathrm{E}_{u, b}\right)
$$

where $P_{u, b}$ is the allocated power of user $k$ in the sub-band $b$, and $\mathrm{E}_{u, b}$ is the effective SINR of user $k$ in this sub-band. The optimization problem can then be formulated as follows

$$
\begin{array}{cl}
\max _{S_{u}} & \sum_{u=U_{Q o s}+1}^{U} \sum_{b \in S_{u}} r_{u, b} \\
\text { subject to } & \sum_{b \in S_{u}} r_{u, b} \geq R_{u}, \quad u=1, \ldots, U_{Q o S} \\
& \sum_{u=1}^{U} \sum_{b=1}^{B} P_{u, b} \leq P_{T}
\end{array}
$$

where $B$ is total number of sub-bands, $R_{u}$ is the QoS users required data rate, $S_{u}$ is the set of sub-bands assigned to user $u$. In our case, $S_{1}, S_{2}, \ldots S_{u}$ are disjoint and each user is assigned one sub-band during one time interval. The formulated problem is a mixed integer programming problem which is hard to solve. However, we can convert this problem into a convex optimization problem by adopting a new parameter $\rho_{u, b}$ as proposed in [12]. It represents a time-sharing factor for the user $u$ of the sub-band $b$. The optimization problem can be reformulated as 


$$
\begin{aligned}
\max _{\rho_{u, b}} & \sum_{u=U_{Q o s}+1}^{U} \sum_{b \in S_{u}} \rho_{u, b} \log _{2}\left(1+\frac{P_{u, b} \mathrm{E}_{u, b}}{\rho_{u, b}}\right) \\
\text { subject to } & \sum_{b=1}^{B} \rho_{u, b} \log _{2}\left(1+\frac{P_{u, b} \mathrm{E}_{u, b}}{\rho_{u, b}}\right) \geq R_{u}, \quad u=1, \ldots . . U_{Q o S} \\
& \sum_{u=1}^{U} \rho_{u, b}=1 \quad \forall b \quad 0 \leq \rho_{u, b} \leq 1 \quad \forall u, b \\
& \sum_{u=1}^{U} \sum_{b=1}^{B} P_{u, b} \leq P_{T}
\end{aligned}
$$

Solving this convex optimization problem using Lagrangian gives the optimal solution

$$
\begin{array}{ll}
\log _{2}\left(\frac{\mathrm{E}_{u, b}}{\gamma \ln 2}\right)-\frac{1}{\ln 2}\left(1-\frac{\gamma \ln 2}{\mathrm{E}_{u, b}}\right)-\beta_{b}=0 & \text { for BE users } \\
\alpha_{u}\left[\log _{2}\left(\frac{\alpha_{u} \mathrm{E}_{u, b}}{\gamma \ln 2}\right)-\frac{1}{\ln 2}\left(1-\frac{\gamma \ln 2}{\alpha_{u} \mathrm{E}_{u, b}}\right)\right]-\beta_{b}=0 & \text { for QoS users }
\end{array}
$$

where $\alpha_{u}, \beta_{b}$ and $\gamma$ are the Lagrange multipliers.

Consequently, using the KKT conditions [13], we conclude the following

$\rho_{u^{\prime}, b}^{*}=1, \quad \rho_{u, b}^{*}=0 \quad$ for all $u \neq u^{\prime}$

where $\mathrm{u}^{\prime}= \begin{cases}\arg \max _{\mathrm{u}}\left(\log _{2}\left(\frac{\mathrm{E}_{u, b}}{\gamma \ln 2}\right)-\frac{1}{\ln 2}\left(1-\frac{\gamma \ln 2}{\mathrm{E}_{u, b}}\right)\right) & \text { for BE users } \\ \arg \max _{\mathrm{u}}\left(\alpha_{u}\left(\log _{2}\left(\frac{\alpha_{u} \mathrm{E}_{u, b}}{\gamma \ln 2}\right)-\frac{1}{\ln 2}\left(1-\frac{\gamma \ln 2}{\alpha_{u} \mathrm{E}_{u, b}}\right)\right)\right) & \text { for QoS users }\end{cases}$

However, finding this optimal solution requires an intensive computation. We need to find the values of $\alpha_{u}$ that satisfy the QoS users constraints regarding the data rate. To do so, an iterative algorithm is defined; we affect small values to $\alpha_{u}$ and increase them one by one to satisfy each QoS user constraint.

\section{CROSS-LAYER SOLUTION}

In order to reduce the complexity of the optimal solution and to exploit all useful information needed for an efficient sub-band allocation, we propose to transform the optimal solution into a simple suboptimal solution that takes into consideration all the required properties and characteristics. 


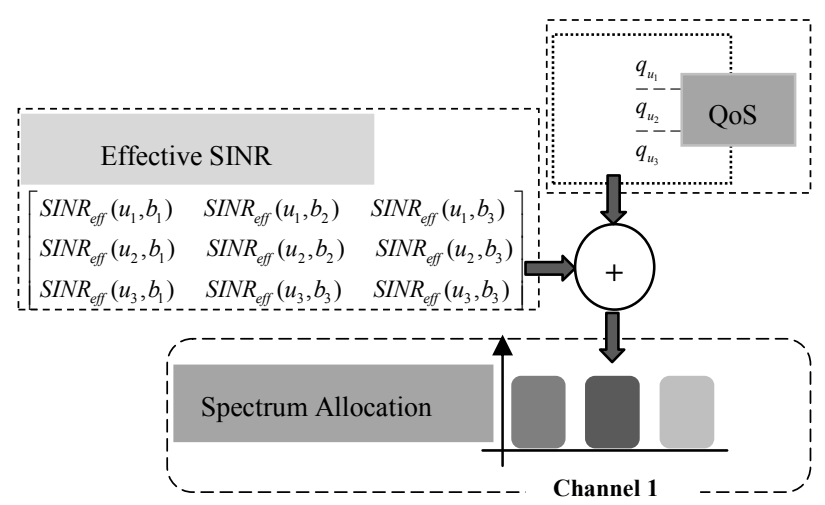

Figure 2. Dynamic cross-layer spectrum allocation design.

Hence, since the complexity of the optimal solution resides in the research of the $\alpha_{u}$ parameter, we propose to study its characteristics in order to find an alternative solution for setting it up. Note that this parameter concerns the QoS users only. Thus, we propose to define a QoS entity that is responsible for affecting a weight or priority level for QoS users. In fact, this entity should be issued from the MAC layer which is charged for QoS support and medium access control. As a result, the proposed cross-layer approach consists in combining the information provided by the PHY layer through the exploitation of the effective SINR and the MAC layer through the weight generator entity (see Fig. 2). Consequently, the most powerful sub-band is assigned to the user $u$ having the highest allocation level $A L$ given by

$$
A L_{u, b}=q_{u}+\max _{b}\left(\operatorname{SINR}_{e f f}(u, b)\right)
$$

where we have a perfect balance between the MAC and the PHY layers.

The advantage of this cross-layer approach is that it is simple to implement and it agrees with the MAC conditions of the system that is based on a distributed architecture where there is no central coordinator for the spectrum management, so that each device is responsible for all computation and measurements for the allocation. Consequently, this new approach provides a low-complexity self-computation mechanism and assumes a decrease in the data exchange between all the users.

\section{SYSTEM PERFORMANCE}

In this section, we present the simulation results for the proposed multiuser cross-layer allocation scheme and we compare the performance of 
the new scheme with that of the optimal solution as well as the single-user WiMedia solution using TFC. Therefore, we use the proposed WiMedia data rates (see Table 1). The results are performed on the first three WiMedia sub-bands (3.1- $4.7 \mathrm{GHz}$ ) for CM1 channel model.

In Fig. 3, the case of three users transmitting simultaneously in the first channel is shown. The three users have different data rates in order to show the advantage of QoS users on BE users in terms of error rate. The QoS user is allocated a high data rate from Table $1(400 \mathrm{Mbit} / \mathrm{s})$ and the BE users are user outperforms the BE users although it is transmitting at a higher data allocated a lower data rate $(320 \mathrm{Mbit} / \mathrm{s})$. As illustrated in the figure, the QoS user outperforms the BE users although it is transmitting at a higher rate.

In Fig.4, we compare the performance of a QoS user transmitting at a rate of $320 \mathrm{Mbps}$ in the cross-layer solution to that in the optimal solution and to the single-user WiMedia solution with TFC. Note that for the single-user solution TFC is exploited because it offers better performance. As shown in the figure, for a $B E R=10^{-4}$, the cross-layer and the optimal solutions are too close and offer a $2.5 \mathrm{~dB}$ gain for the QoS user compared to WiMedia solution.

In Fig.5, we consider the case of BE users transmitting at a rate of 200 $\mathrm{Mbit} / \mathrm{s}$ and present their performance in the optimal and cross-layer solutions. For a $B E R=10^{-4}$, the optimal solution offers a $0.5 \mathrm{~dB}$ compared to the cross-layer solution. On the other hand, we note that the performance of the cross-layer in the case of BE users is close to that of the single-user WiMedia solution. This proves that the performance of the multiuser crosslayer solution performance is never degraded compared to the single-user WiMedia solution.

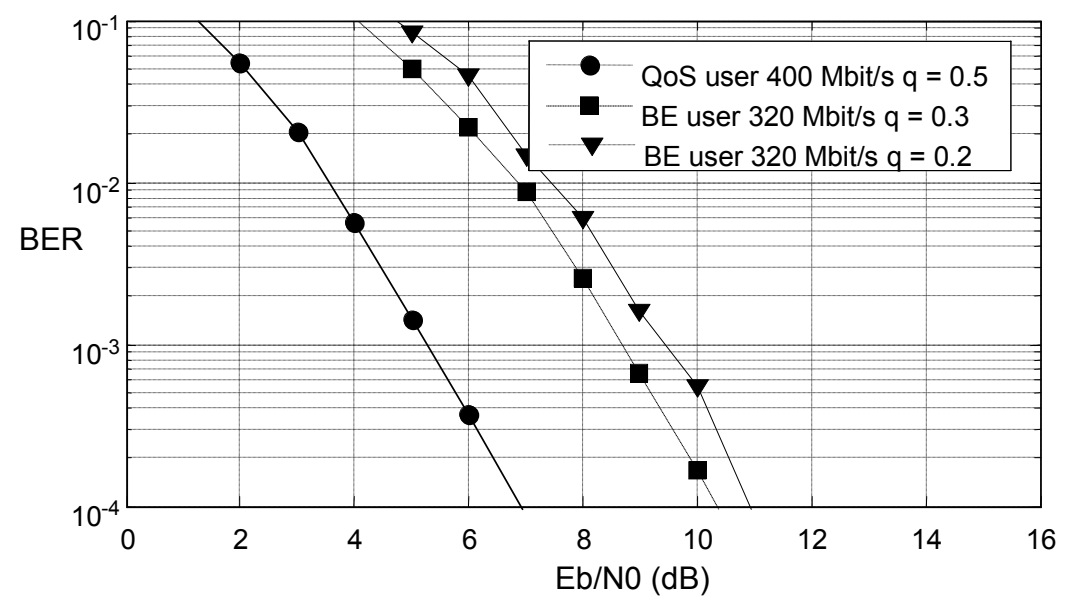

Figure 3. Three users performance in the cross-layer solution. 


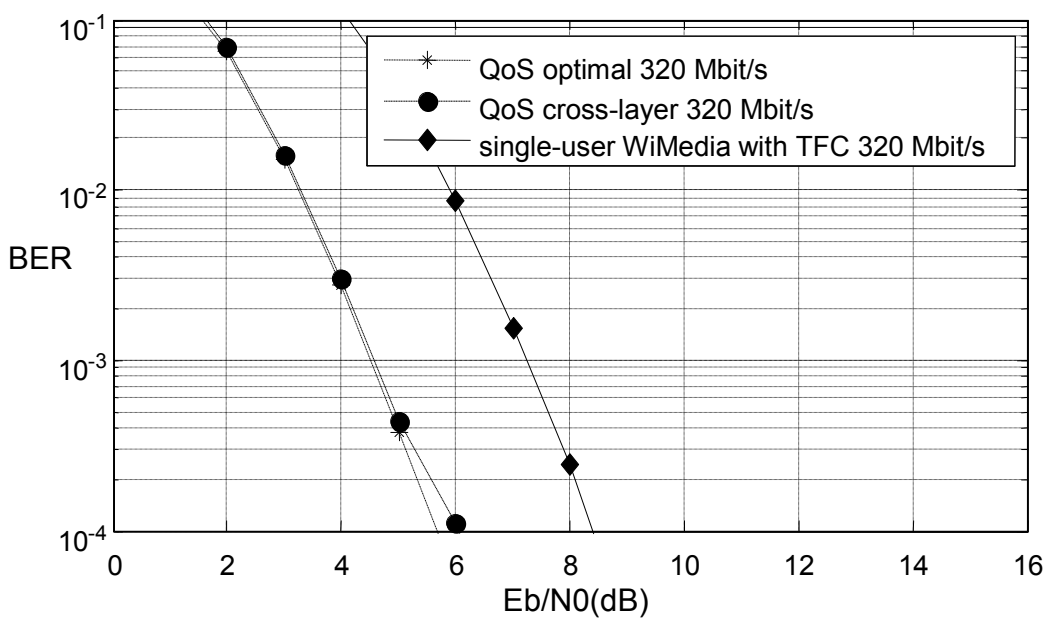

Figure 4. Performance comparison of QoS users.

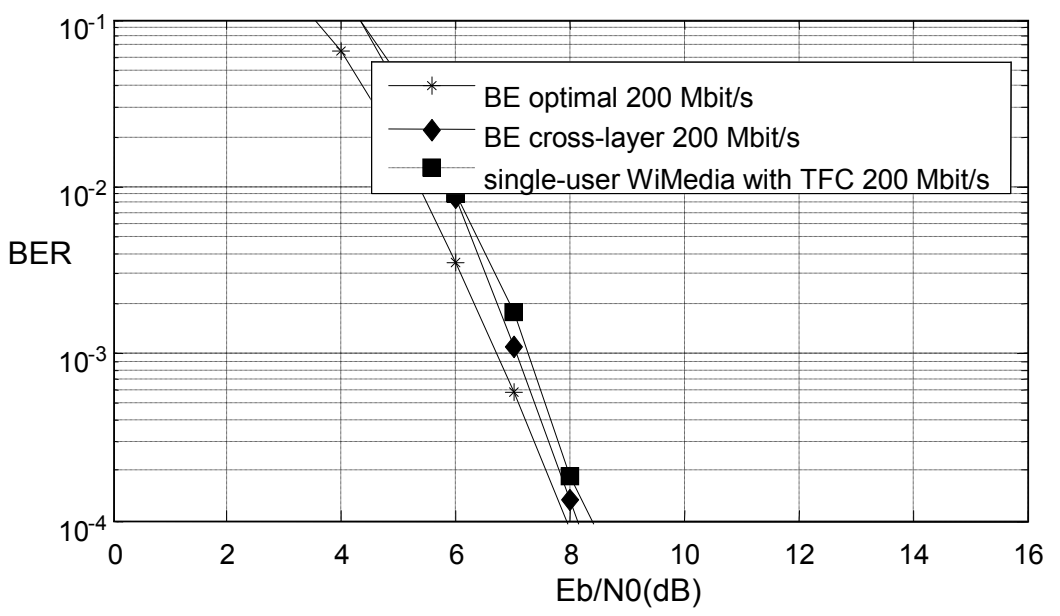

Figure 5. Performance comparison of BE users.

\section{CONCLUSION}

In this work, we proposed a dynamic cross-layer design for UWB systems. This design combines the physical layer information through the investigation of the effective SINR and the MAC layer through the QoS parameters. We studied first the optimal solution of the dynamic allocation under QoS constraints by exploiting the effective SINR and formulating an optimization convex problem. By analyzing the properties of the optimal solution we obtained, we proposed a lower complexity solution that is based on a cross-layer approach by defining a simple linear allocation function. 
Simulation results showed that the new multiuser cross-layer approach outperforms the WiMedia solution proposed for a single-user scheme. Moreover, we showed that the cross-layer solution performance is close to the optimal solution performance.

\section{ACKNOWLEGMENT}

The research leading to these results has received funding from the European Community's Seventh Framework Programme FP7/2007-2013 under grant agreement $\mathrm{n}^{\circ} 213311$ also referred as OMEGA.

\section{REFERENCES}

[1] "First report and order, revision of part 15 of the commission's rules regarding ultra-wideband transmission systems," FCC, ET Docket 98-153, Feb. 14, 2002.

[2] Batra et al.. "Multi-Band OFDM physical layer proposal for IEEE 802.15 task group 3a," IEEE document P802.15-04/0493r1, Texas Instruments et al., Sept. 2004.

[3] WiMedia Alliance, Inc., "Multiband OFDM physical layer specification," Release 1.1, July 2005.

[4] Standard ECMA-368, High rate ultra wideband PHY and MAC standard, $2^{\text {nd }}$ edition, September 2007.

[5] Z. Chen, D. Wang and G. Ding, "An OFDM-UWB Scheme with Adaptive Carrier Selection and Power Allocation," in Proc. IEEE Intern. Conference on Wireless Communications, Networking and Mobile Computing (WiCOM '06), pp.1-4, China, Sept. 2006.

[6] A. Stephan, J-Y. Baudais and J-F. Helard, "Efficient Allocation Algorithms for Multicarrier Spread Spectrum schemes in UWB applications," in Proc. IEEE Intern. Conference on Ultra-Wideband (ICUWB '07), pp. 551-555, Singapore, Sept 2007.

[7] W.P Siriwongpairat, Z. Han and K. J Ray Liu, "Power controlled channel allocation for multi-user multiband UWB systems," IEEE Trans. Wireless Communications, vol. 6, no. 2, pp. 583-592, Feb. 2007.

[8] 3GPP TSG-RAN-1, "R1-030999: considerations on the system-performance evaluation of HSDP using OFDM modulations"; RAN WG1 \#34.

[9] 3GPP TSG-RAN-1, "R1-040090: system level performance evaluation for OFDM and WCDMA in UTRAN".

[10] B. Muquet, Z. Wang, G-B Giannakis, M. de Courville and P. Duhamel, "Cylic pefix or zero padding for wireless multicarrier transmission," IEEE trans. Communications, vol. 50, pp. 2136-2148, Dec. 2002 .

[11] J. Foester, "Channel Modeling sub-committee report (final)," IEEE P802.15.-02/490rl-SG3a,2003.

[12] P. W. C. Chan and R. S. Cheng, "Optimal power allocation in zero-forcing MIMO-OFDM downlink with multiuser diversity," in Proc. of IST Mobile \& Wireless Communications Summit, Dresden, June 2005.

[13] D.P Bertsekas, Nonlinear Programming $2^{\text {nd }}$ Edition. Athena Scientific, 1999. 